\title{
Nano Sensors and Devices for Space and Terrestrial Applications
}

\author{
Jing Li ${ }^{1}$, Dan Powell ${ }^{2}$, Stephanie Getty ${ }^{2}$, Yijiang $\mathrm{Lu}^{3}$ \\ ${ }^{1}$ NASA Ames Research Center \\ ${ }^{2}$ NASA Goddard Space Flight Center \\ ${ }^{3}$ Eloret Corporation \\ Coordinated by: Dan Powell, NASA Goddard Space Flight Center
}

\section{Abstract}

The chemical sensor market has been projected to be $\$ 40$ billion dollars worldwide in less than 10 years. The development of chemical sensors is to monitor and control environmental pollution, to improve the diagnostics for point care in medical applications, to provide small, low power, fast, and sensitive tools for process and quality control in industrial applications, and to implement or improve detection of warfare threats and security. In all these applications, there is a demand for improved sensitivity, selectivity and stability beyond what is offered by commercially available sensors. It is believed that the emerging field of nanotechnology can play an important role in realizing these goals, and the proposed concept represents a significant step in that direction.

Nanotechnology provides the ability to work at the molecular level, atom by atom, to create large structures with fundamentally new molecular organization. It is essentially concerned with materials, devices, and systems whose structures and components exhibit novel and significantly improved physical, chemical and biological properties, phenomena, and processes due to their nanoscale size.

A nanosensor technology has been developed in NASA Ames using nanostructures, such as single walled carbon nanotubes (SWNTs) and metal oxides nanobelts or nanowires, on a pair of interdigitated electrodes (IDE) processed with a silicon-based microfabrication and micromachining technique. The IDE fingers were fabricated using photolithography and thin film metallization techniques. Both in-situ growth of nanostructure materials and casting of the nanostructure dispersions were used to make chemical sensing devices. These sensors have been exposed to nitrogen dioxide, acetone, benzene, nitrotoluene, chlorine, and ammonia in the concentration range of $\mathrm{ppm}$ to $\mathrm{ppb}$ at room temperature. The electronic molecular sensing of carbon nanotubes in our sensor platform can be understood by electron modulation of the nanostructured devices and analytes in terms of charge transfer mechanisms. As a result of the charge transfer, the conductance of nanostructures will change. The metal oxide nanobelts sensors operate at much lower temperature around $150^{\circ} \mathrm{C}$ compared with $500^{\circ} \mathrm{C}$ for conventional metal oxides sensors with same sensing behavior. Due to the large surface area, low surface energy barrier and high thermal and mechanical stability, nanostructured chemical sensors potentially can offer higher sensitivity, lower power consumption and better robustness than the state-of-the-art systems, which make them more attractive for defence and space applications, and for other commercial applications. Combined with MEMS technology, light weight and compact size sensors can be made in wafer scale with high yield and low cost. Additionally, a wireless capability of such a sensor chip can be used for networked mobile and fixed-site detection and warning systems for military bases, facilities and battlefield areas. 


\section{Rationale for Recommendation}

Chemical sensors have a wide application spectrum from space mission to homeland security and other commercial applications. Chemical sensor market is steadily increased and projected to be $\$ 40$ billion US dollars in next 10 years.

The nanosensor and nanodevice based on nanostructures are recommended here for possible commercialization. The research effort on this technology has been funded by NASA and FAA with interagency agreement in past several years. The development of recommended nanosensor and nanodevices has gained NASA's attention for funding in future years to raise the technical readiness level (TRL) for space mission. Currently, NASA is putting $\$ 500-\$ 600 \mathrm{~K}$ in this project to develop a sensor module that has a sensor chip contains 32 sensing channels using different nanostructured materials, a complete electronic system for sensing signal acquisition, and a pneumatic pathway for gas sample delivery. This sensor module will be plugged in a satellite secondary payload in an orbit around $500 \mathrm{~km}$ for a flight demonstration of trace chemical detection using nanosensors. A prototype can be easily derived from this flight module.

The research results show that our nanosensors provide high sensitivity (ppm-ppb), low power consumption ( $\mu \mathrm{W}-\mathrm{mW})$, compact in size and mass, easy integration to the existing electronic system (e.g. flight demo unit will have power and data communication pins in one connector to interface with satellite's main board). With the optimized combination of different nanostructures in a sensor array, selectivity for detection of complex chemical mixtures or discriminate the different chemical environments will be improved. Nanotechnology allows working at atomic and molecular level, which enables us to miniaturize the sensor device and make a high density sensor array that can greatly increase the detection capability by using the redundant sensing elements, and design an orthogonal sensor array with different nano materials. Fabrication process has been developed for large scale production. The recommended nanosensor is illustrated below:
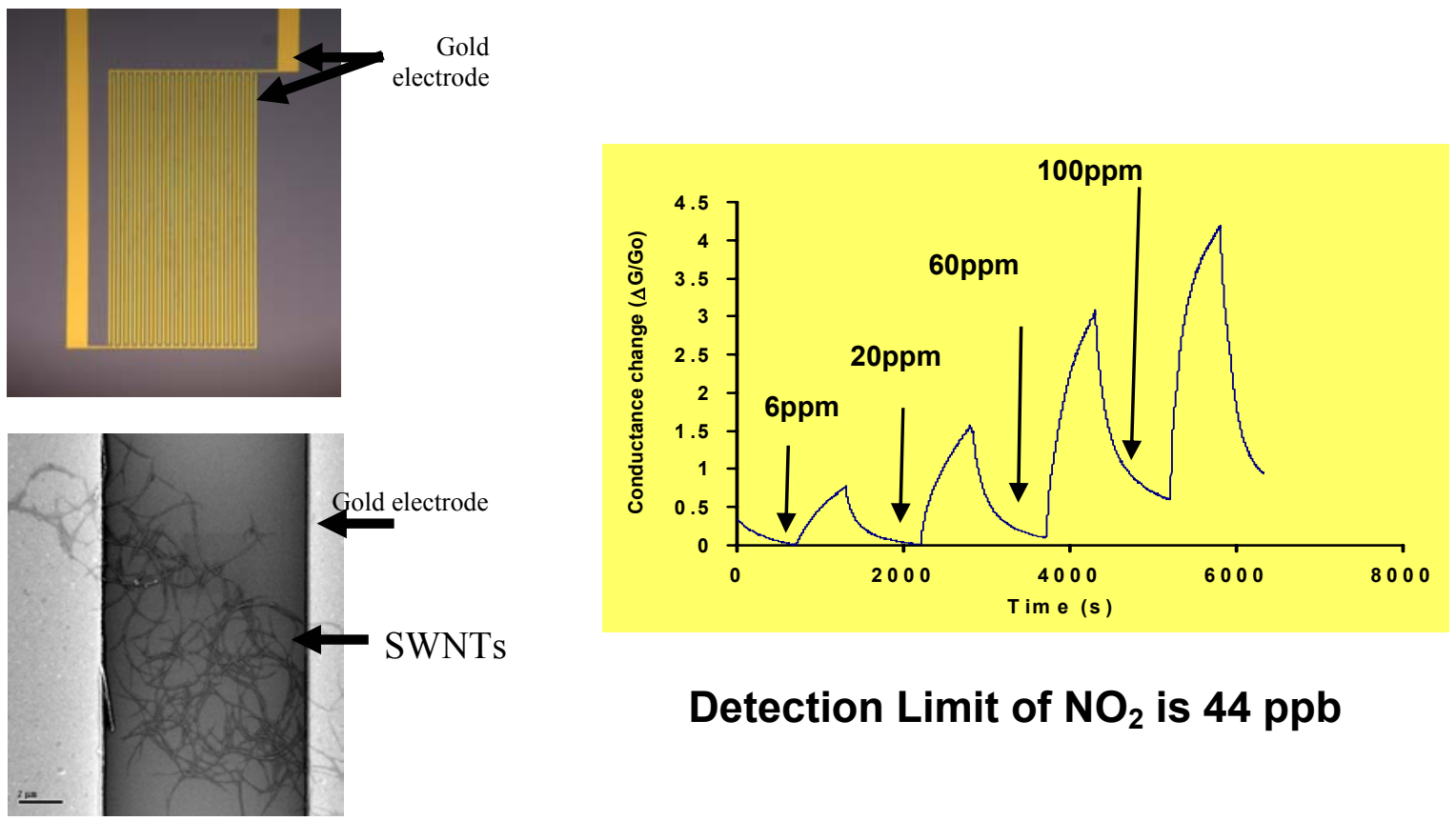

\section{Detection Limit of $\mathrm{NO}_{2}$ is $44 \mathrm{ppb}$}

Figure 1Nanosensor and its response to $\mathrm{NO}_{2}$ at different concentrations. Image of interdigitated electrode (upper left); Carbon nanotubes across gold electrodes (lower left); sensor response curve to NO2 (right). 


\section{Current State-of-the-Art}

Nanotechnology provides the ability to work at the molecular level, atom by atom, to create structures with fundamentally new molecular organization. Nanostructured materials such as carbon nanotubes (CNT) offer superior performance over conventional approaches due to the remarkable mechanical properties and unique electronic properties as well as the high thermal and structural stability. Single-walled carbon nanotubes (SWNT) have all the atoms on the surface that are exposed to the environment, allowing a change in physiochemical properties sensitively. The lower energy barrier on the surface of CNT ensures the room temperature sensing, which allow much lower power consumption in $\mu \mathrm{W}$ to $\mathrm{mW}$ per sensor. They have great potential for developing a new generation of chemical sensors to detect gas and vapor species. Carbon nanotube-based chemical sensors have the following properties:

- High sensitivity (potentially single molecule sensitivity) due to large surface to volume ratio,

- Fast response due to the one-dimensional quantum wire nature that makes its electronic properties very sensitive to gas adsorption,

- Lower power consumption (at least 100 times less than the current system), which is ideal for persistent surveillance applications,

- Small size and lightweight.

Our design of the carbon nanotube-based chemical sensor uses an interdigited electrode platform, and purified single wall carbon nanotubes (SWCNTs) based materials for chemical sensing. Unlike the recent attempts that involved a field effect transistor approach with CNT as the conducting channel, our platform is essentially a chemiresistor. Here, we demonstrate a simple SWNT sensor platform that combines the advantages of both single nanotube transistors and the film based nanotube sensors with extended applications to sensitive detection of organic vapors. In this platform, SWNTs form a network or mesh on interdigitated electrodes (IDE) using a solution casting process (see Figure 1) providing a large enough density of nanotubes for sensor performance. The IDE configuration enables effective electric contact between SWNTs and the electrodes over large areas while providing good accessibility for gas/vapor adsorption to all SWNTs including semiconducting tubes. This approach provides much simpler fabrication process with high yield of nanosensors that are robust, reliable, and reproducible in their sensor performance. The relatively simple fabrication is important to developing inexpensive sensor systems for the cost-conscious chemical sensor market. Above all, this type of sensor is amenable to integrate into a system.

Our preliminary work with CNTs indicates a conductivity change with exposure to $\mathrm{NO}_{2}$, nitrotoulene (stimulant of explosives), a variety of other organic and inorganic vapors and gases. Preliminary results also show sensitivity in the ppb range with reproducibility of sensitivity around $6 \%$ sensor to sensor. By loading the CNTs with catalytic metal nano clusters and coating polymers on the surface of CNT, great selectivity was achieved for room temperature detection of methane (combustible) in low ppm and chlorine (toxic) gas in ppb level.

Based on this nanosensor technology, a sensor module with a 32-channel array loaded with different nanostructures is under development for a space flight demonstration experiment. This sensor module will be in a satellite and fly in the mid orbit around 500kM for trace chemical detection.

Combining the carbon nanotube-based chemical sensor with MEMS technology, a lab-ona-chip can be built, which has onboard data processing and potentially wireless communication capabilities. Such a lab-on-a-chip system can be deployed across a wide spectrum of hardware platforms for environmental monitoring, and can be made into a portable handheld device. 
Concept Paper Title

\section{Baseline Use}

A recent report to the President recommends redoubling efforts to improve long-term climate modeling. NASA's Earth Science Enterprise's (ESE) action plan includes uninhabited aircraft measurements of greenhouse gases to validate satellite observations. Also, the president announced a new vision in January for NASA to extend human presence in solar system. Smart detection and monitoring is one of the challenges that NASA is focusing on through the current effort on the human and robotic technology for space exploration. This requires the development of miniature detectors - which exhibit selectivity, sensitivity, and time response equal to or better than current state of the art sensor technology - that are compact, robust and have low mass \& power budgets.

This proposed task will develop the miniature, chemical sensor systems which have multiple uses in power systems, propellant production, and planetary chemical analysis. A broad based, one-stop-sensor array meeting a broad range of needs is the objective of this sensor system. This group is leading in the development of micro, nano sensor technology for toxic gas and volatile organic vapors detection. The prototype Nanosensor unit for flight demonstration in space is progressively developed and will deliver to Navy for a satellite launch in 2006.

The product of our proposed work not only has the potential use in space systems, but also has a wide spectrum of terrestrial applications. Some of these include: hydrogen sensors for fuelcell applications; carbon monoxide and NOx sensors for emissions monitoring; ammonia and perfluoropropane in refrigerant leak monitoring; and carbon monoxide, ozone, and VOC sensors for air quality monitoring. The high performance sensors developed will be suitable for use in hostile environments and are amenable to miniatureization (both inherent benefits of solid-state sensors over most other gas sensor technologies). The benefits to the commercial market are to solve several key market needs including increased sensitivity, higher reliability, and possibly longer times between calibrations. Sensor users are constantly looking for technologies that are low-cost and enhanced stability, lower detection limits and lower operating power, all potential improvements with the proposed technology.

The immediate use of this product is in defense arena for hazardous gases and vapors detection. The Department of Defense has identified a requirement for 270,000 JCAD (Joint Chemical Agent Detector) systems. The JCAD is a small, lightweight, high-performance chemical detection system that offers networked or stand-alone chemical detection. It can be hand-held or operated from a vehicle or from a fixed installation. Our sensing platform fits right there in this application.

\section{Trends Impacting Improvement}

Advancement in several areas can directly impact the improvement of our nanosensor technology. They are: 1) Nanostructured materials development; 2) high speed computing capability; 3) Nano printing and inkjetting technologies development; 4) wireless and network capability.

Nanostructured materials: Nanostructured materials have unique structural properties, such as high surface area, single crystalline, and well organized molecular structure. These properties are critical to the gas sensing due to the adsorption process prior to the chemical to electrical signal conversion. High surface area ensures high sensitivity, single crystalline provides stability of the nanostructures for sensor stability, and well organized molecular structure can assure the reproducibility of sensors' performance.

High speed computing capability: With the advance of the computing and computer technology, fast data acquisition, transformation for gathering transient chemical signal is possible. This will 
Concept Paper Title

provide a platform to advance the sensing technology from data collection, signal processing, and information transformation point of view.

Nanoprinting and nanoinkjetting: Currently, there are many efforts on nano printing and nano inkjetting technology to get more precise positioning with less and small size of deposition spots. The development in this area will impact our nanosensors for scale up in mass production with automation capability and easy quality control process.

Wireless and network technology: Taking the advantages of wireless and networking capability, our nanosensors can extend the market from existing point care or measurement to a network mapping measurement. For example, the nanosensors can be used for environmental and safety monitoring each room and hallway in buildings.

\section{Alternative Approaches and Organizations}

The challenges in this technology are the selectivity of the sensors and the recovery time for sensor reuse. Alternative approaches can be taken to improve and ensure this technology leading the state of the art. The alternative approaches can be classified as 1) sensing materials, 2) sensing platforms.

Sensing materials: Semiconductor nanowires have been the subject of enthusiastic research efforts to enable a large variety of compositions, to incorporate dopants, and to investigate the promise of these structures to field-effect transistor, chemical and biological sensor, and optoelectronic technologies. Although many of these characteristics have been the subject of proof-of-principle experiments, much work remains until semiconductor nanowires can be integrated into a commercially viable technology.

It is within the scope of this concept paper to suggest future integration of this alternative inorganic approach into the fully developed interdigitated electrode array.

Sensing platforms: In analogy to the bulk properties, unadulterated nanowires have been demonstrated to function as switches, diodes, and photonic nanodevices. Related devices have been fabricated to demonstrate the sensitivity of these semiconductor nanostructures to chemical environment. Drawing on the principles of electrostatic gating, polar and charged analytes can be detected as a change in device conductivity, sensitivity to atmospheric gases has been investigated and various examples of biological sensing have been achieved.

Sensing platform, also called transducer, can influence the sensor performance as well. One platform has its own advantages over the others. The effect of sensing platform is also sensing material dependent. A good coupling of sensing material with platform will enable high sensitivity and selectivity with their specific features, such as gating effect of transistors, thermal power of chemically sensitive thermisters, impedence change of capacitors and Shottky diodes. A hybrid sensing platform can be composed to optimize the detection capability using sensor array approach with the advantages of each platform.

Another approach can be used in sensing platform alternation is the high density sensor array. Our nano sensor can be self-assembled to a high density $\left(10^{3}-10^{6} / \mathrm{cm}^{2}\right)$ array with low power consumption (at least 100 times less than the current system), light and compact (at least 10 times smaller than the current system), and with proved high sensitivity (ppb-ppt), accuracy and rapid response. Inputs from high density nanosensor arrays will provide a high discrimination power that can accurately monitor and understand the chemical environment in region and allow appropriate response on the part of the crew or vehicle system. It can also be used for homeland security and battle field chemical weapon detection. 


\section{Leading Aerospace Applications}

There are two leading aerospace applications. One is to greatly increase the science measurement capability with compact size, less mass and power requirements for electronics and sensors; to provide a portable device for composition measurements of Earth's atmosphere; to make highly miniaturized gas detectors enabling Earth Science Enterprise Plans for in situ measurements to validate satellite observations. Another application is to deploy the nanosensors which ensure the proper operation in the microgravity or reduced gravity environment, especially those which must operate in multiphase media, or are strongly impacted by the lack of natural buoyancy, such as combustion and precombustion monitors. The unique microgravity considerations make this deliverable particularly NASA-specific. Microgravity transport modeling capability will be employed to enable interpreration of sensor data, such as localization of hazards. Sample acquision for accurate monitoring must account for the complexities of multiphase (gas, liquid, solid) behavior in micro or reduced gravity, and must also require little or no crew time and expendables. Sample handling may be necessary to achieve the necessary sensitivity and selectivity. The recommended nanosensors can be used for External Environment Monitoring to monitor hazardous conditions in the extra-vehicular environment. Hazards in these regions include but are not limited to reactive chemicals, erosive dust, and radiation.

\section{Potential Non-Aerospace Applications}

The proposed technology has a specific application in space mission. Meanwhile it has a wide spectrum of application in terrestrial area, such as defense, industry, environmental, and medical and biological applications.

Industry: Nanostructure based chemical sensors possess high sensitivity, small size and low power consumption, which can be used to quickly verify incoming raw materials at the delivery point; to monitor the changing composition of the vapor phase surrounding or contained within the process. Much like vision inspection is used to assess the visual integrity (color, shape, size) of products, olfactory inspection assesses the chemical integrity (consistency, presence of contaminants). The technology can significantly reduce the amount of time and money spent analyzing those materials in a lab, as well as reducing the amount of materials handling.

Environment: Increasing awareness and new regulations for safety and emission control make environmental monitoring one of the most desired amongst the numerous industrial and civil applications for which the development of reliable solid-state gas sensors is demanded. Current methods for air quality control approved by the standards consist of analytical techniques, which need the use of very costly and bulky equipment. For applications in this arena, sensors that are able to selectively detect various gases at a concentration level of a few ppb and in the form of lowcost portable handheld devices for continuous in-situ monitoring are needed. With unique advantages of high sensitivity, small size and low power consumption, and strong mechanical and thermal stability, carbon nanotube based chemical sensors are best fit for this type of application. Defense: Chemical sensors are very focused for security and defense applications due to their portability and low power consumption. Carbon nanotube sensors potentially can offer higher sensitivity and lower power consumption than the state-of-the-art systems, which make them more attractive for defense applications. Some examples include monitoring filter breakthrough, personnel badge detectors, embedded suit hermiticity sensors and other applications. Additionally, a wireless capability with the sensor chip can be used for networked mobile and fixed-site detection and warning systems for military bases, facilities and battlefield areas.

Medical/Bio: It is believed that chemical sensors would provide physicians with a quicker and more accurate diagnostic tool. Applications could include obtaining objective information on the identity of certain chemical compounds in exhaled air and excreted urine or body fluids related to 
Concept Paper Title

specific metabolic conditions, certain skin diseases or bacterial infections, such as those common to leg or burn wounds. Additionally, the chemical sensors may provide more accurate, real-time patient monitoring during anaesthesia administration.

It is note worth that the sensing platform developed in this project can also be used in liquid phase for heavy metals and $\mathrm{pH}$ detection, as well as for bio species detection, such as pathogen, bacteria, and enzymes.

\section{Drivers for Change}

Chemical sensors currently on the market already demonstrate the necessity for high surface area for analyte capture. Surface roughening is employed in various approaches to enhance surface area. One consequence of this approach is the associated compromise of electronic properties upon the introduction of disorder. The preservation of crystalline order that coexists with the inherent surface area enhancement of organic and inorganic nanowires illustrates one of their major advantages.

The high operating temperatures requisite to conventional metal oxide gas sensors also suggest an area of improvement. In fact, our preliminary investigations into nanostructured metal oxide sensors $(\mathrm{ZnO})$ suggest that operating temperatures are significantly reduced upon reduction to the nanoscale. The cylindrical configuration of these prototypical devices indicates that surface energy is an important parameter in operating temperature considerations.

Surface energy is intimately tied to surface structure, and further techniques to modify surface structure can be investigated through the concept of functionalization that can also improve the selectivity.

\section{Recommended Approach(es)}

The scope of possibilities that is demonstrated by these early devices suggests a significant payoff for future development. Our extensive knowledge of the surface chemistry of nanostructures combine with simple sensing platform, IDE, suggests the potential for a large assortment of near-term applications. The recommended approaches for advancing our nanosensor technology are:

1. Functionalization of nanostructures to improve the specificity;

2. Doping the nanostructures with catalysts to improve sensitivity, selectivity, and fast response time;

3. Nanostructures/polymer composites to make low cost, easy fabrication sensor arrays;

4. Hybrid sensing platform to maximize the array performance;

5. Temperature cycling to enhance the recovery time and improve the selectivity.

\section{Rationale for Recommendations}

Above recommended approaches are commonly used in commercial sensor technologies. Our design of the sensing platform using interdigitated electrode is mature and easy to work with. The sensing material is the key to the chemical sensing. Nanostructured materials provide not only the unique physiochemical properties in nanoscale, but also provide a well organized structure atom by atom for us to manipulate the surface and bulk structures. This gives us a lot of room to play with and enhance the sensor performance through the modification and stimulation of nanostructured materials.

The technical team has variety knowledge background in chemistry, physics, materials science, analytical instrumentation and nanotechnology. 


\section{Summary of Prototype Identification and potential Implementation Plan}

The practical objectives of this development are:

- Design and fabricate nano structure engineered chemical sensor array with 32 channels. (year 1-2)

- Design, fabrication and tests of a static system for Lab test (year 1).

- Testing of an evolved static system at a range of atmospheric pressures in a chamber readily available for field test. (year 1-2)

- Design and fabrication of a prototype of sensor array chip for multi purpose use. (year 2)

- Design and fabricate a prototype of a handheld device for demonstration in field test. (year 3)

Specific objectives and milestones are as follows:

- Improve sensor sensitivity and response time to gases and vapors (e.g. NOx, toluene, etc),

- Investigate materials and techniques appropriate to enhance sensor selectivity and stability

- Study environmental effects, such as humidity, temperature and altitude/pressure, for sensor performance

- Optimize sensor design and the electrode/transducer platform process

- Investigate the impact of data processing protocols to the precision and accuracy of chemical detection

- Demonstrate a proper handheld device for in-situ chemical detection

\section{Potential Investment Possibilities and Risk}

This nanosensor technology is readily for mass production in commercial market. The prototype of the nanosensors can be easily derived from the sensor module that is currently under development for a flight demonstration experiment and that will be delivered to the satellite carrier by end of February 2005.

With the technology readiness and a wide spectrum of applications, potential investment possibilities can be from venture capitals, industry partners, and government contracts.

Risk: 1) intellectual properties management; 2) infrastructure for scale up production; 3) resources assurance.

\section{References:}

1. Yijiang Lu, Jing Li, Jie, Han, H-T Ng, Christie Binder, Christina Partridge, M. Meyyappan, Accepted by Chemical Physics Letters.

2. Jing Li, Chemical and Physical Sensors in Carbon Nanotubes: Science and Applications, Editor: M. Meyyappan, CRC Press, Boca Raton, FL, USA, 2004.

3. Marin Cinke, Jing Li, Charles Bauschlicher, Alessandra Ricca, M. Meyyappan, Chemical Physics Letters, 376, 761-766, 2003.

4. Jing Li, Yijiang Lu, Qi, Ye, Martin Cinke, Jie Han, and M. Meyyappan, Nanoletters, 3, No.7, 929-923, 2003.

5. Martin Cinke, Jing Li, Bin Chen, Alan Cassell, Lance Delzeit, Jie Han and M. Meyyappan, Chemical Physics Letters, 365, 69-74, 2002.

6. Brain Matthews, Jing Li, Steven Sunshine, Lee Lerner, and Jack Judy, IEEE Sensor Journal, Special Issue on Electronic Nose Technologies, Vol. 2, No. 3, 160-168, 2002. 
7. X. Duan, Y. Huang, and C. M. Lieber, Nano Letters 2, 487 (2002).

8. H. T. Ng et al., Applied Physics Letters 82, 2023 (2003).

9. Y. Cui et al., Science 293, 1289 (2001).

10. D. Zhang et al., Applied Physics Letters 83, 1845 (2003).

11. O. H. Elibol et al., Applied Physics Letters 83, 4613 (2003).

12. J. Hahm and C. M. Lieber, Nano Letters 4, 51 (2004).

13. C. Li et al., Applied Physics Letters 83, 4014 (2003). 